\title{
H/D Exchange Studies in Metabolite Identification
}

\section{Raju Bandu* and Kwang Pyo Kim}

Department of Applied Chemistry, Kyung Hee University, Yongin City, Republic of Korea

Hydrogen/deuterium (H/D) exchange LC-MS experiments play an important role in identification and structure elucidation of metabolites. $\mathrm{D}_{2} \mathrm{O}$ as sheath liquid and deuterated solvents are used as a mobile phase in deuterated LC-MS experiments for the analysis of metabolites. Deuterated experiments are useful in the structural characterization of metabolites by determining the numbers and positions of exchangeable protons present in different functional groups such as $-\mathrm{NH}-,-\mathrm{NH}_{2},-\mathrm{OH},-\mathrm{COOH}$ and $-\mathrm{SH}$ in various metabolites [1,2]. The mass shift of the deuteriated molecular ion from that of the protonated molecular ion gives information about the total number of exchangeable protons present in the structures of metabolites [3-6]. The structure elucidation of unknown metabolites can be effectively carried out by using deuterated precursor ions compared to protonated precursor ions where tandem MS spectra of deuterated metabolites help to determine the exact location of the exchanged protons $[3,5]$. It can be noted that the online deuterated experiments are useful to characterize the metabolites without the use of large scale preparation for other analytical characterizations. Further, the deuterated experiments allowed to distinguish the hydroxylated and glucuronide metabolites from other metabolites with the help of numbers of exchangeable hydrogen's $[3,5]$. Moreover, deuterated experiments also allowed to distinguish the metabolites that have the same nominal mass and identical elemental compositions which may not be possible by normal LC-MS/MS [5]. In conclusion, deuterated studies can be used to further support the structural confirmation of metabolites irrespective of biological matrices.

\section{References}

1. Lam W, Ramanathan R (2002) In electrospray ionization source hydrogen/ deuterium exchange LC-MS and LC-MS/MS for characterization of metabolites. J Am Soc Mass Spectrom 13: 345-353.

2. Liu DQ, Hop CE (2005) Strategies for characterization of drug metabolites using liquid chromatography-tandem mass spectrometry in conjunction with chemical derivatization and on-line H/D exchange approaches. J Pharm Biomed Anal 37: 1-18.

3. Raju B, Ramesh M, Borkar RM, Srinivas R, Padiya R, et al. (2012) In vivo metabolic investigation of moxifloxacin using liquid chromatography/ electrospray ionization tandem mass spectrometry in combination with online hydrogen/deuterium exchange experiments. Rapid Commun Mass Spectrom 26: 1817-1831.

4. Raju B, Ramesh M, Borkar RM, Padiya R, Banerjee SK et al. (2012) Identification and structural characterization of in vivo metabolites of ketorolac using liquid chromatography electrospray ionization tandem mass spectrometry (LC/ESI-MS/MS). J Mass Spectrom 47: 919-931.

5. Bandu R, Ahn HS, Lee JW, Kim YW, Choi SH, et al. (2015) Liquid Chromatography Electrospray Ionization Tandem Mass Spectrometric (LC/ESIMS/MS) Study for the Identification and Characterization of In Vivo Metabolites of Cisplatin in Rat Kidney Cancer Tissues: Online Hydrogen/Deuterium (H/D) Exchange Study. PLoS One 10: e0134027.

6. Raju B, Hak Jin K, Hyuck Jun M, Kwang Pyo K (2015) A liquid chromatography electrospray ionization tandem mass spectrometric study (LC/ESI-MS/MS) of in vivo metabolites of cisplatin in rat liver and brain tissues: deuterated experiments. RSC Adv 5: 89951-89958.
${ }^{*}$ Corresponding authors: Bandu R, Department of Applied Chemistry, College of Applied Science, Kyung Hee University, Yongin City, Republic of Korea, Tel: +82-10-4042-8806; E-mail: raju.bandu@gmail.com

Kim KP, Department of Applied Chemistry, College of Applied Science, Kyung Hee University, Yongin City, Republic of Korea, Tel: +82-31-201-5260; E-mail: kimkp@khu.ac.kr

Received June 23, 2016; Accepted June 24, 2016; Published June 27, 2016

Citation: Bandu R, Kim KP (2016) H/D Exchange Studies in Metabolite Identification. Metabolomics 6: e149. doi:10.4172/2153-0769.1000e149

Copyright: $\odot 2016$ Bandu R, et al. This is an open-access article distributed under the terms of the Creative Commons Attribution License, which permits unrestricted use, distribution, and reproduction in any medium, provided the original author and source are credited. 